\title{
Overexpression of Dlx2 leads to postnatal condyle degradation
}

\author{
JIEWEN DAI, JIAWEN SI, XIAOFANG ZHU, LEI ZHANG, DANDAN WU, \\ JINGTING LU, NINGJUAN OUYANG, XUDONG WANG and GUOFANG SHEN
}

\author{
Department of Oral and Cranio-Maxillofacial Surgery, Shanghai Ninth People's Hospital, \\ Shanghai Jiao Tong University School of Medicine, Shanghai Key Laboratory of Stomatology, Shanghai 200011, P.R. China
}

Received July 11, 2015; Accepted May 31, 2016

DOI: $10.3892 / \mathrm{mmr} .2016 .5406$

\begin{abstract}
Distal-less homeobox 2 (Dlx2), a member of the Dlx family of transcription factors, is important for the development of craniofacial tissues. Previous studies based on knock-out mutant mice revealed that Dlx2 primarily disturbed the development of tissues from maxillary arch. The present study used a transgenic mouse model to specifically overexpress Dlx2 in neural crest cells in order to investigate the role of Dlx2 overexpression in post-natal condyle in mice. The model was constructed and the phenotype observed using gross observation, micro-CT scan and histological examination. The model determined that overexpression of Dlx2 may lead to postnatal condyle malformation, subchondral bone degradation and irregular histological structure of the condylar cartilage. In addition, the expression of osteocalcin in the condyle region was markedly downregulated, whereas expression of msh homeobox 2 was upregulated. The results of the present study suggest that Dlx2 overexpression in cranial neural crest cells would disrupt the development of post-natal condyle, which demonstrates that the expression level and the spatiotemporal expression patterns of Dlx2 may be important in regulating the development of post-natal condyle in mice, and also offered a possible temporal-mandibular joint osteoarthritis model animal for future studies.
\end{abstract}

\section{Introduction}

The distal-less homeobox (Dlx) gene family contains six members (Dlx1-6), which are organized into three convergent pairs (Dlx1/2, Dlx $3 / 4$ and Dlx $5 / 6$ ), located on chromosome 2 in mice, and exhibit a nesting expression pattern in the first and second branchial arch region $(1,2)$. Dlx 1 and 2 and are expressed

Correspondence to: Dr Guofang Shen or Professor Xudong Wang, Department of Oral and Cranio-Maxillofacial Surgery, Shanghai Ninth People's Hospital, Shanghai Jiao Tong University School of Medicine, Shanghai Key Laboratory of Stomatology, 639 Zhizaoju Road, Shanghai 200011, P.R. China

E-mail: maxillofacsurg@163.com

E-mail: xudongwang70@hotmail.com

Key words: overexpression, Dlx2, post-natal, condyle, degradation in the epithelium and the cranial neural crest cells (CNCCs) derived from mesenchyme in the maxillary and mandibular processes $(2,3)$. Previous studies based on knock-out mutant mice revealed that a null mutant of Dlx2 may lead to malformation in craniofacial tissues, including maxilla, nasal bone, auditory ossicle and maxillary teeth. The 'Dlx family' regionalize the jaw primordium with Dlx1/2 regulating upper jaw development and Dlx $5 / 6$ regulating the development of the lower jaw (2,4-8). A previous study also determined that an overexpression mutant of Dlx2 leads to sorting and aggregation of CNCCs (9). Additionally, it has been demonstrated that Dlx2 overexpression may result in ectopic skeletal and cartilaginous elements, in the maxillary and mandibular region in ovo (10). Our previous study also revealed that overexpressing Dlx2 in neural crest cells (NCCs) in mice, resulted in midfacial clefts, nasal and premaxillary hypoplasia and spinal deformities (11).

The mandibular condyle in mammals is a primary site of mandible growth and a major element of the temporal-mandibular joint (TMJ) $(12,13)$. The mandibular condylar cartilage is ontogenetically characterized as a secondary cartilage, and is important for the development and function of TMJ (14). Although numerous factors are involved in the development of the mandibular condyle and certain genes have been previously demonstrated to have interactions with the Dlx gene family $(14,15)$, to the best of our knowledge, the exact molecular mechanisms regulating the emergence and cellular organization of the condylar cartilage and the degradation of condylar cartilage remain to be fully elucidated.

Regarding the effects of overexpression of Dlx 2 in the craniofacial region, previous studies in virus-infected ovo or transgenic mice have determined that the overexpression of Dlx2 primarily affects the development of tissues from the maxillary arch $(10,11)$. The comprehensive effects of overexpression of Dlx2 on mammalian mandibular condyle remains to be fully elucidated. The aim of the present study was to assess the effects of overexpression of Dlx 2 on postnatal condyle development.

\section{Materials and methods}

Mouse strains. Dlx2 C57BL/6J genetic background conditional overexpression transgenic mice (iZEG-Dlx2) were bred in Department of Oral and Cranio-Maxillofacial Surgery, Shanghai Ninth People's Hospital (Shanghai, China), and 
Wnt1-Cre transgenic mice were obtained from the Jackson Laboratory (Bar Harbor, Maine, USA). iZEG-Dlx2 transgenic mice were further bred with Wnt1-Cre transgenic mice to obtain the mice, which specifically overexpress Dlx2 in NCC derived tissues (Wnt1Cre::iZEG-Dlx2), as previously described (Fig. 1) (11). All animal experimental procedures were performed in compliance with the guidelines of the National Institute of Health in the United States and Institutional Animal Care and Use Committees of the Shanghai Ninth People's Hospital, Shanghai Jiao Tong University School of Medicine, and were reviewed and approved by the Ethical Committees of the Shanghai Ninth People's Hospital, Shanghai Jiao Tong University School of Medicine, Shanghai, China (approval no, 2013-7).

Mandible preparations. The mice (age, 90 days; mean weight, $25.2 \mathrm{~g}$ ) were housed in a pathogen-free condition with a $14 / 10$-h light/dark cycle, in conditions of $18-23^{\circ} \mathrm{C}$ and $40-60 \%$ humidity. Mice were euthanized by carbon dioxide. All mice were used for analysis regardless of gender. The mandibles of eight P90 Wnt1Cre::iZEG-Dlx2 and control mice were separated from the surrounding tissue, and were transferred to $95 \%$ ethanol for $48 \mathrm{~h}$. The tissues were subsequently stained with Alizarin red and Alcian blue, as previously described (11). Subsequently, the stained/unstained condyle was transferred to a mixed solution of glycerol and water (50:50) for image capturing under an integrated microscope.

Histological analysis. The condyles of P90 Wnt1Cre::iZEG-D1x2 and control mice were dissected, followed by fixation in $4 \%$ paraformaldehyde for $24 \mathrm{~h}$ at room temperature and demineralization in 0.5 M EDTA for 14-20 days also at room temperature. The tissues were embedded in paraffin with a section at thickness of $5 \mu \mathrm{m}$, then stained with hematoxylin and eosin (H\&E) or Alizarin red/Alcian blue, as previously described (11), followed by mounting with resinous mounting medium for image capturing on an integrated microscope.

Micro-computed tomography (CT) scans. The skulls/mandibles of the P90 control and Wnt1Cre::iZEG-Dlx2 mice were fixed with $4 \%$ paraformaldehyde at room temperature for $24 \mathrm{~h}$. Next, the tissues were scanned using an eXplore Locus MicroCT scanner (GE Healthcare Life Sciences, Milwaukee, WI, USA). The slice thickness used for micro CT scans was $10 \mu \mathrm{m}$. Reconstruction of 3D skulls and bone mineral density (BMD) calculations were performed using GE MicroView software version 2.2 (GE Healthcare Life Sciences).

Immunohistochemistry. The condyles of the P90 control and Wnt1Cre::iZEG-D1x2 mice were demineralized and embedded in paraffin, and sectioned at a thickness of $5 \mu \mathrm{m}$. The immunohistochemistry process was performed as previously described $(11,16)$. Briefly, antigen retrieval for slides was performed with a bone antigen restoration liquid kit (Sunteam Biotech Co., Ltd. , China) and subsequently, the samples were blocked for 60 min with $3 \%$ bovine serum albumin in phosphate-buffered saline (PBS) containing $0.2 \%$ Triton-X-100. Subsequently, the sections were incubated with anti-Dlx2 (1:100; Abcam, Cambridge, UK; cat. no. ab18188), anti-osteocalcin (OCN; 1:200; Abcam; cat. no. ab93876) or anti-msh homeobox 2 (Msx2; 1:100; Abcam; cat. no. ab69058) overnight at $4^{\circ} \mathrm{C}$. Following incubation, donkey anti-rabbit secondary antibodies (AlexaFluor 488-conjugated; Thermo Fisher Scientific, Inc.; cat. no. A21206) were diluted in PBS (1:300) and incubated for $60 \mathrm{~min}$ at room temperature. Finally, the slides were mounted with Vectashield mounting medium containing 4',6-diamidino-2-phenylindole (Invitrogen; Thermo Fisher Scientific, Inc.) was used to mount the coverslips. Images were capture under a fluorescence microscope.

Statistical analysis. All data were expressed as the mean \pm standard deviation. The difference between experimental groups was assessed by independent Student's t-test using SPSS software version 18.0 (IBM SPSS, Chicago, IL, USA). P<0.05 was considered to indicate a statistically significant difference.

\section{Results}

Establishment of WntlCre::iZEG-Dlx2 mouse strain. Dlx2 (C57BL/6J genetic background) conditional overexpression transgenic mice (iZEG-Dlx2) were bred in our laboratory. The iZEG-Dlx2 transgenic mice were mated with Wnt1-Cre transgenic mice to obtain mice, which specifically overexpress Dlx2 in NCC-derived tissues (Wnt1Cre::iZEG-Dlx2). This was performed as previously described in detail (Fig. 1) (11).

Morphology of condyles of P90 Wnt1Cre::iZEG-Dlx2 mice. Observation under an integrated microscope demonstrated that P90 Wnt1Cre::iZEG-D1x2 mice exhibited smaller mandible and condyle, and the condylar cartilage cap was malformed and thinner compared with the control group (Fig. 2). At P90, the reconstructed 3D images from micro-CT highlighted a cross-bite malocclusion, and bone defect in the mandible of Wnt1Cre::iZEG-Dlx 2 mice (Fig. 3A and B). The 3D images and sagittal sections also showed osteoporosis in the condylar bone when compared with the control group (Fig. 3C and D). BMD analysis based on micro-CT examination suggested a significant decrease in BMD in the condylar bone in P90 Wnt1Cre::iZEG-Dlx2 mice compared with control mice $(\mathrm{P}<0.01$; Fig. 3E). This demonstrated that osteoporosis occurred in the condyle, and implied that overexpression of Dlx2 may lead to condyle degradation or abnormal development.

Histological appearance of condyle of P90 WntlCre::iZEG-Dlx2 mice. The condyle of P90 Wnt1Cre::iZEG-Dlx2 mice was analyzed in detail. H\&E and Alizarin red/Alcian blue staining of coronal sections indicated that the Wnt1Cre::iZEG-Dlx2 mice exhibited an irregular condylar cartilage structure and a uneven condylar cartilage surface. Condylar cartilage were disorganized and narrower, irregular in size and lacked a regular arrangement of four layers, including resting layer, proliferative layer, hypertrophic layer and mineralized zone. Additionally, less hypertrophic chondrocytes were observed in the condylar cartilage in P90 Wnt1Cre::iZEG-Dlx2 mice (Fig. 4). Bone loss was also evident in the condylar bone region when compared with the control group (Fig. 4). The phenotype demonstrated was observed in six condyle from three mice. Although the 
iZEG-Dlx2 transgenic mouse

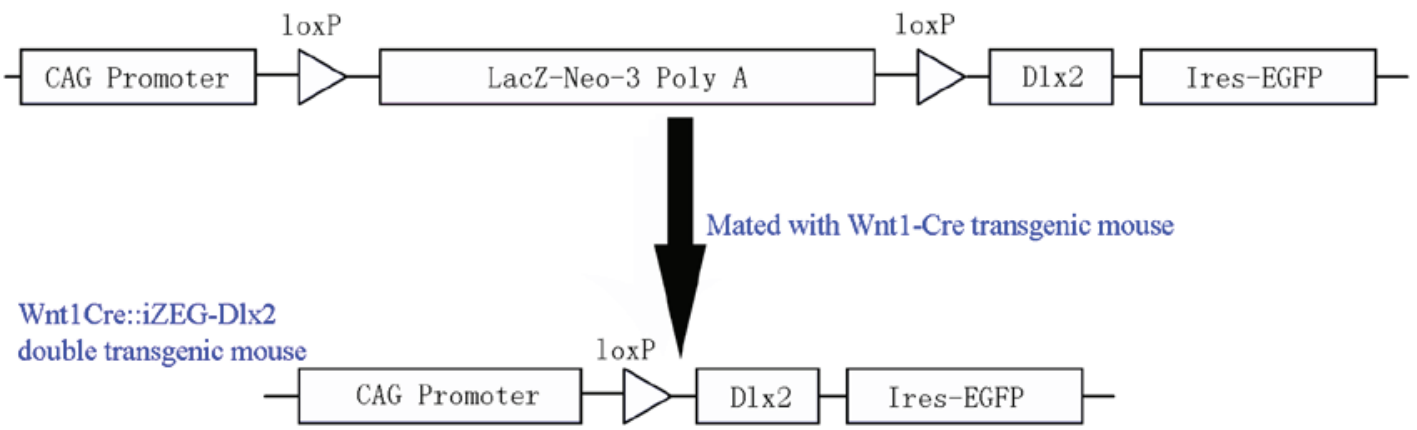

Figure 1. Schematic representation of the Cre-mediated excision of the LacZ-Neo-STOP cassette in Wnt1Cre::iZEG-Dlx2 mice when iZEG-Dlx2 mice were mated with Wnt1-Cre mice.
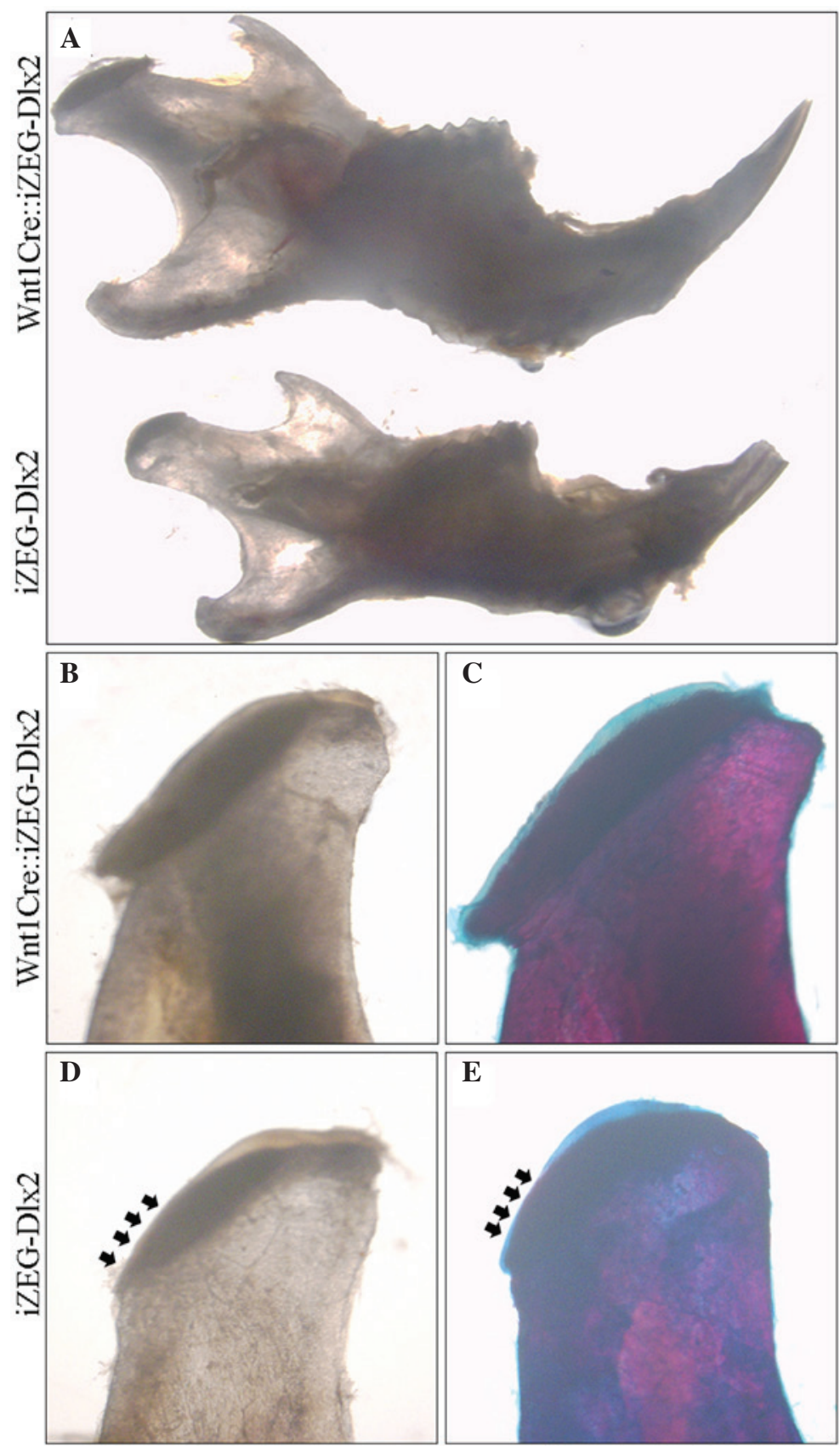

Figure 2. Overexpression of Dlx2 leads to postnatal condyle dysmorphia. (A) Small and abnormal condyle in P90 Wnt1Cre::iZEG-Dlx2 mice. (B and D) and Alizarin red/Alcian blue staining ( $\mathrm{C}$ and $\mathrm{E}$ ) under an integrated microscope showed a thin cartilage cap (denoted with arrow heads) in the condyle in P90 Wnt1Cre::iZEG-Dlx2 mice. Dlx2, distal-less homeobox 2. 
A

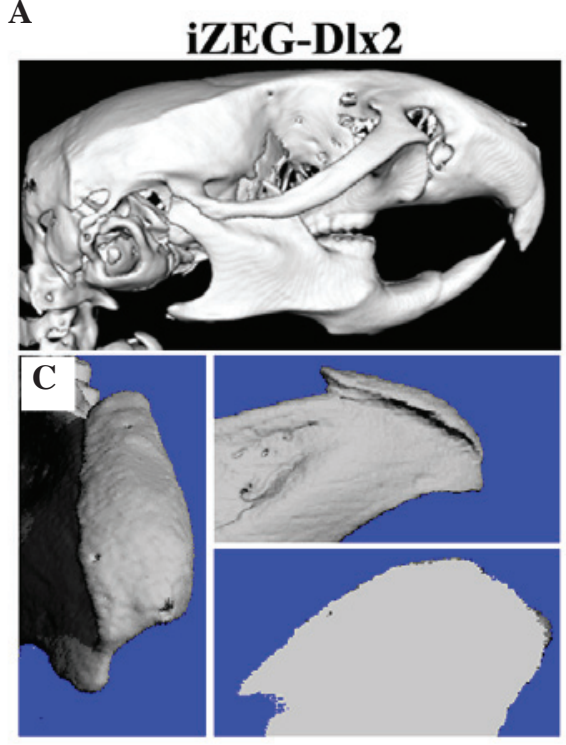

B
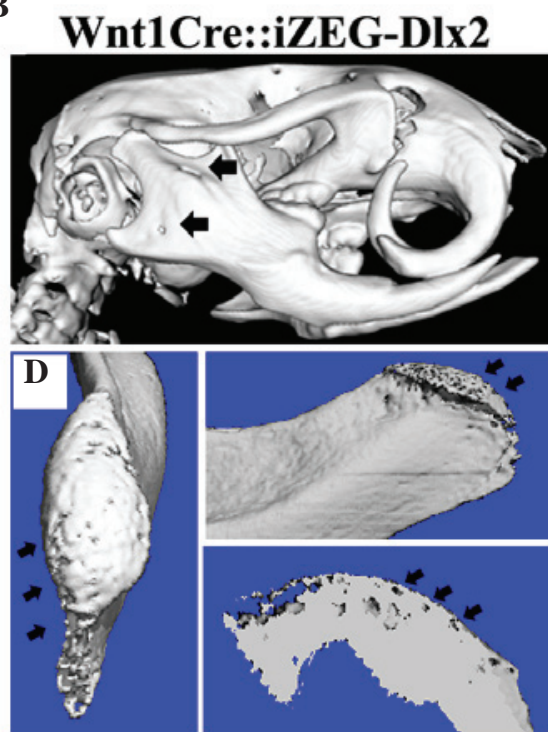

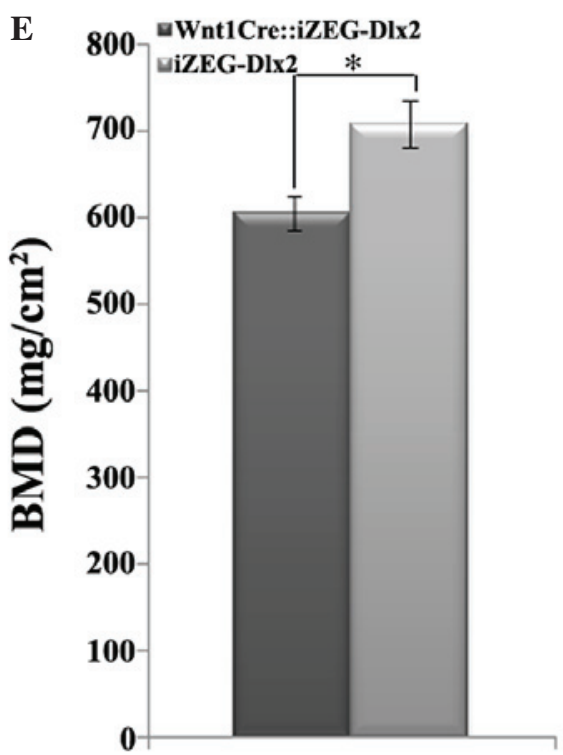

Figure 3. Micro-CT examination. (A and B) CT scan of iZEG-Dlx 2 mice and the P90 Wnt1Cre::iZEG-Dlx2 mice, respectively. Cross-bite malocclusion and defect in mandibular ramus (arrow head) was observed in the latter. (C and D) 3D and sagittal sections of iZEG-Dlx2 mice and the P90 Wnt1Cre::iZEG-Dlx2 mice, respectively. Condyle osteoporosis (arrow head) was observed in the latter. (E) BMD analysis, based on micro-CT examination, revealed a significant decrease in BMD in condyle in P90 Wnt1Cre::iZEG-Dlx 2 mice. The data are presented as the mean \pm standard deviation ( $\mathrm{n}=3$; ${ }^{*} \mathrm{P}<0.01 \mathrm{vs}$. the.iZEG-Dlx2 mice). Dlx2, distal-less homeobox 2; BMD, bone mineral density.

three mice exhibited similar phenotype, the severity was not consistent in each mouse; therefore, the expression pattern of Dlx2 may not be the same across the different mice.

Expression of Dlx2, OCN and Msx2 in the condylar region in P90 WntlCre::iZEG-Dlx2 mice. Immunofluorescence staining also indicated that Dlx 2 expression levels were higher in the condylar region in P90 Wnt1Cre::iZEG-Dlx2 mice (Fig. 5A and B). The expression levels of OCN, a marker of osteogenesis, were downregulated in the condylar cartilage and bone region in P90 Wnt1Cre::iZEG-Dlx2 mice (Fig. 5C and D). Therefore, it is possible that osteogenesis has been impaired in the condylar region. The expression of Msx2, important for craniofacial bone development, which had been identified to interact with Dlx2 in previous studies (17-20), was upregulated in the condylar region, particularly in the resting layer and mineralized zone (Fig. 5E and F). Dlx 2 may interact with Msx2 in the process of condyle development or degradation.

\section{Discussion}

Previous studies have determined that inactivation of Dlx 1 and Dlx 2 results in defects in the upper jaw, whereas inactivation of Dlx 5 and Dlx6 leads to homeotic transformation of the lower jaw into an upper jaw (2). Therefore, the Dlx family may regionalize the jaw primordium, and Dlx1/2 regulate upper jaw development, whereas Dlx5/6 control the lower jaw (6-8). The present study was the first, to the best of our knowledge, to describe the effect of overexpression of Dlx 2 on postnatal condyle degradation, and revealed that the Dlx 2 overexpression in NCCs in mice may lead to postnatal condyle 


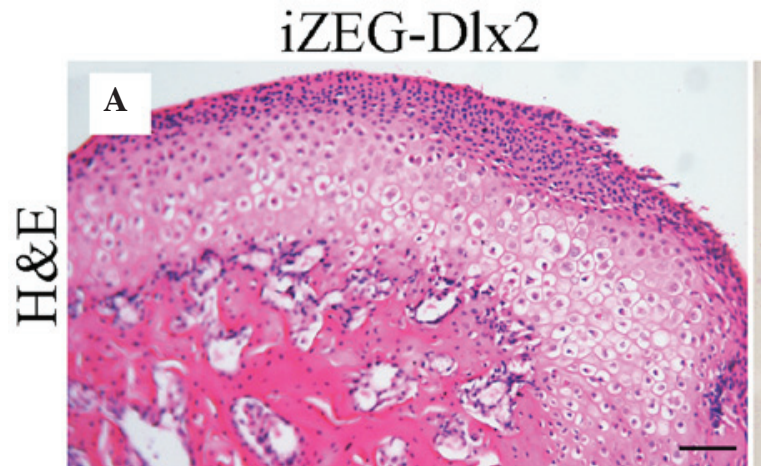

Wnt1Cre::iZEG-Dlx2
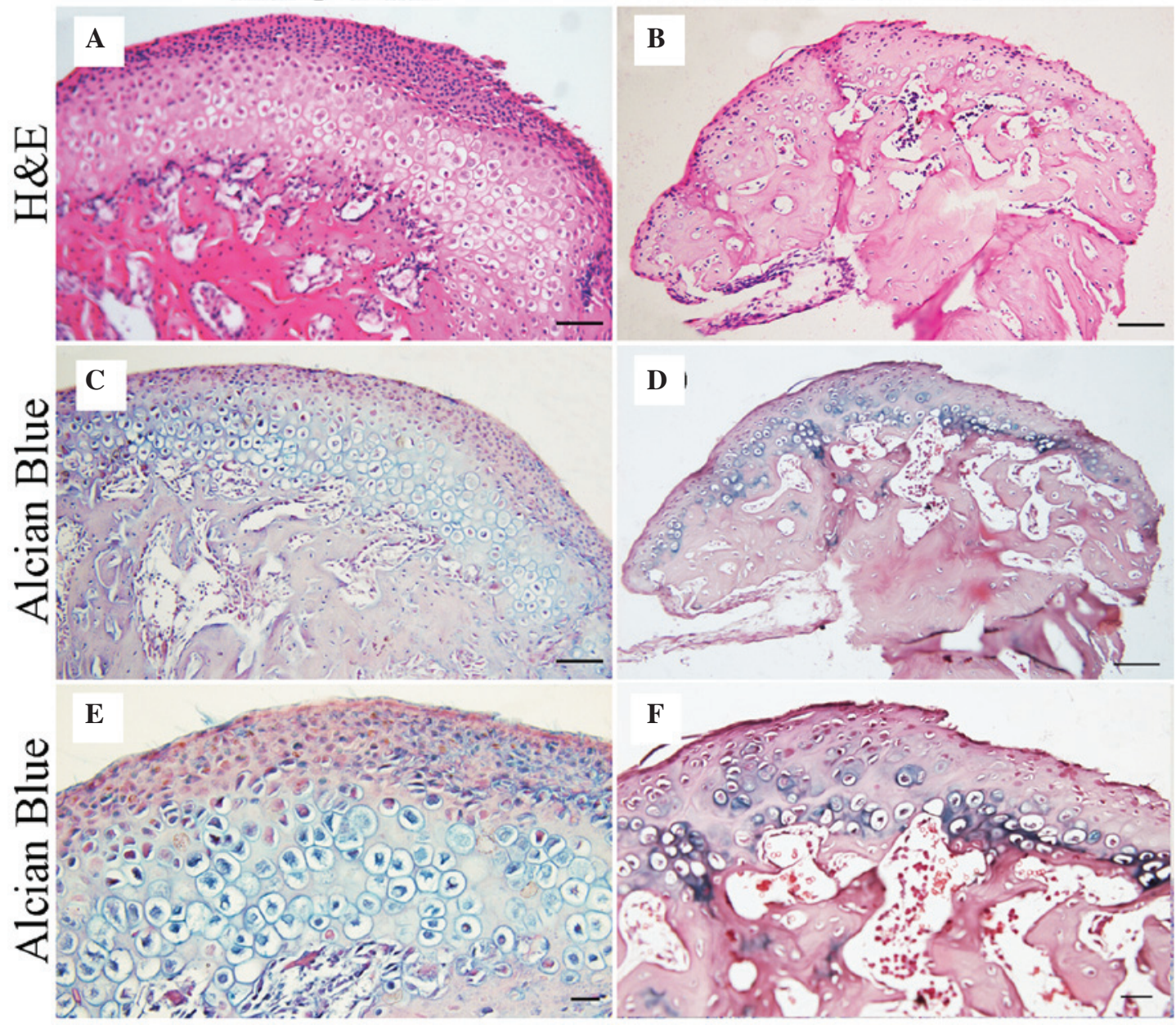

Figure 4. Histological analysis of condyle in P90 Wnt1Cre::iZEG-Dlx2 mice. (A and B) Sagittal sections and H\&E staining of iZEG-Dlx2 mice and P90 Wnt1Cre::iZEG-Dlx2 mice sections (Scale bar, $100 \mu \mathrm{m}$ ). Subchondral bone degradation and irregular cartilage histological structure was observed in the latter. (C-F) Sagittal sections and Alizarin red/Alcian blue staining showed irregular cartilage histological structure and less hypertrophic chondrocytes (blue) in the P90 Wnt1Cre::iZEG-Dlx2 mice. (E and F) Magnification corresponding to regions in C and D, respectively (Scale bar, $50 \mu \mathrm{m}$ ). H\&E, hematoxylin and eosin; Dlx2, distal-less homeobox 2 .

malformation and osteoporosis. The present study revealed that Dlx2 had effect on the lower jaw. It is possible that the Dlx $1 / 2$ vs. Dlx $5 / 6$ proteins exert unique roles in specifying maxilla and mandible (qualitative hypothesis), whereas higher levels of total Dlx protein in mandible can disrupt the development of condyle (quantitative hypothesis) (6). Previous studies also revealed that Dlx6 activity in lower jaw development is partially shared by Dlx $1 / 2$. Additionally, Dlx $1 / 2$ function is partially redundant with Dlx $5 / 6$ in regulating mandibular gene expression, which may further support the findings of the present study (6). The differences in expression level, pattern or timing in the transgenic mice may also contribute to this phenotype.

In condyles overexpressing Dlx2, an irregular histological structure was observed; the resting layer was thinner and more likely to undergo premature maturation and hypertrophy. As a consequence, condylar morphology and growth are limited, possibly due to reduced chondroprogenitor cell proliferation and impaired osteogenesis. Lower expression levels of OCN in condyle of the mice model overexpressing Dlx2 confirm this hypothesis, which is similar to Ihh and Cilia mutant mice $(17,18)$. Our previous study also determined that overexpression of Dlx2 disturbs the nasal bone/cartilage maxilla development, and leads to irregular histological structure of the spinal cartilage (11). In addition, previous studies have demonstrated that Dlx2 overexpression may result in ectopic skeletal and cartilaginous elements in the maxillary and mandibular region in ovo (10). Taken together, it may be suggested that Dlx2 is important for the development of bone and cartilage.

The present study also indicated an upregulation of Msx 2 in the condylar region of Wnt1Cre::iZEG-Dlx2 mice, which was similar to our previous findings in the craniofacial region (11). Previous studies revealed that Msx2 is a downstream gene of Dlx2, and heterodimerization of Msx2 with Dlx2 results in functional antagonism. In addition, Msx2 is important for craniofacial development, and overexpression of Msx2 impedes osteoblast differentiation and triggers multiple craniofacial defects (19-21). Therefore, overexpressed Dlx2 may interact with Msx 2 to regulate the condyle degradation.

Osteoarthritis (OA), typically characterized as progressive cartilage degradation and subchondral bone changes, is a severe pathological change in the condyle of patients with severe temporomandibular disorder (TMD) $(22,23)$. Previous studies 


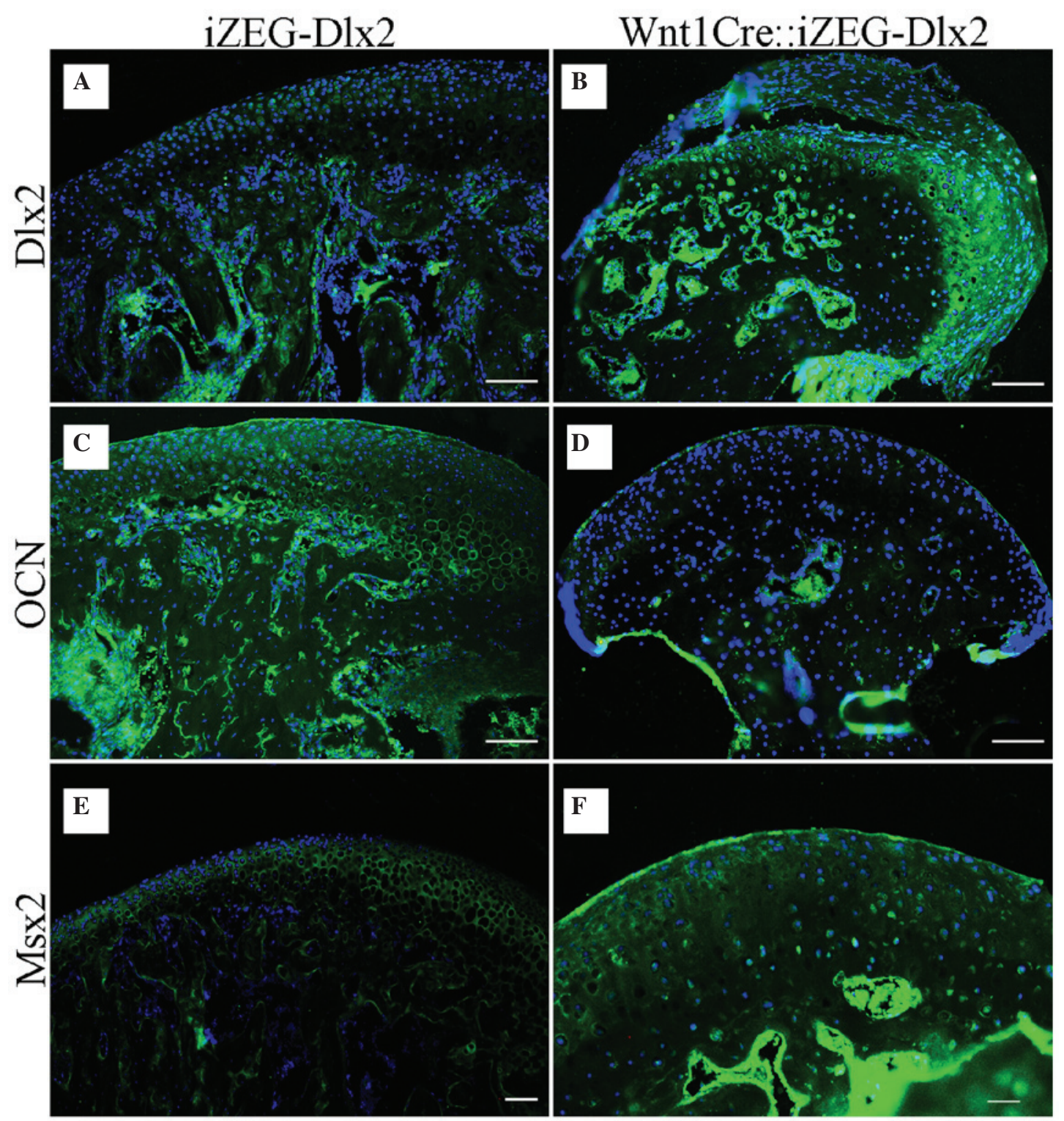

Figure 5. Expression of genes involved in condyle development in P90 Wnt1Cre::iZEG-Dlx2 mice. Immunofluorescence staining showed the expression level of the Dlx2 protein in the condylar region in (A) iZEG-Dlx2 and (B) P90 Wnt1Cre::iZEG-Dlx2 mice (Scale bar, $100 \mu \mathrm{m}$ ). OCN protein expression in the condylar region in (C) iZEG-Dlx2 and (D) P90 Wnt1Cre::iZEG-Dlx2 mice (Scale bar, $100 \mu \mathrm{m}$ ). Msx2 protein expression level in the condylar region of (E) iZEG-Dlx2 and (F) P90 Wnt1Cre::iZEG-Dlx2 mice (Scale bar, $50 \mu \mathrm{m}$ ). Dlx2, distal-less homeobox 2; OCN, osteocalcin; Msx, msh homeobox.

have indicated that low BMD and increased bone turnover in the early stages of OA in the knee joint, and the inhibition of bone resorption for treating OA, which implied that abnormal subchondral bone remodeling is crucial for the development of OA $(23,24)$. In the present study, the Dlx2 overexpressed postnatal condyle resulted in degraded cartilage and subchondral bone osteoporosis, which was similar to a previous study that reported OA transgenic mice overexpressed transforming growth factor- $\beta$ in the subchondral bone (24). Therefore, the present findings implied that overexpression of Dlx2 may contribute to TMJ OA, and present a possible TMJ OA model for future studies.

In conclusion, Dlx2 overexpression in NCCs may lead to postnatal condyle malformation, subchondral bone degradation and irregular histological structure of condylar cartilage. Additionally, Dlx 2 overexpression affected postnatal mandible development and may be used in future TMJ OA model animal studies. The exact molecular mechanisms underlying the effect observed in the present study must be investigated in the future.

\section{Acknowledgements}

The present study was supported by the National Nature Science Foundation of China (nos. 81300842 and 81271122), Emerging Cutting-Edge Technology Joint Research Project of Shanghai Municipal Hospital (no. SHDC12013103) and the Program for Innovation Research Team of Shanghai Municipal Education Commission.

\section{References}

1. Panganiban G and Rubenstein JL: Developmental functions of the Distal-less/Dlx homeobox genes. Development 129: 4371-4386, 2002. 
2. Depew MJ, Simpson CA, Morasso M and Rubenstein JL: Reassessing the Dlx code: The genetic regulation of branchial arch skeletal pattern and development. J Anat 207: 501-561, 2005.

3. Thomas BL, Liu JK, Rubenstein JL and Sharpe PT: Independent regulation of Dlx2 expression in the epithelium and mesenchyme of the first branchial arch. Development 127: 217-224, 2000.

4. Qiu M, Bulfone A, Martinez S, Meneses JJ, Shimamura K, Pedersen RA and Rubenstein JL: Null mutation of Dlx-2 results in abnormal morphogenesis of proximal first and second branchial arch derivatives and abnormal differentiation in the forebrain Genes Dev 9: 2523-2538, 1995.

5. Qiu M, Bulfone A, Ghattas I, Meneses JJ, Christensen L, Sharpe PT, Presley R, Pedersen RA and Rubenstein JL: Role of the Dlx homeobox genes in proximodistal patterning of the branchial arches: Mutations of Dlx-1, Dlx-2, and Dlx-1 and -2 alter morphogenesis of proximal skeletal and soft tissue structures derived from the first and second arches. Dev Biol 185: 165-184, 1997.

6. Jeong J, Li X, McEvilly RJ, Rosenfeld MG, Lufkin T and Rubenstein JL: Dlx genes pattern mammalian jaw primordium by regulating both lower jaw-specific and upper jaw-specific genetic programs. Development 135: 2905-2916, 2008.

7. Beverdam A, Merlo GR, Paleari L, Mantero S, Genova F, Barbieri O, Janvier P and Levi G: Jaw transformation with gain of symmetry after Dlx5/Dlx6 inactivation: Mirror of the past? Genesis 34: 221-227, 2002

8. Depew MJ, Lufkin T and Rubenstein JL: Specification of jaw subdivisions by Dlx genes. Science 298: 381-385, 2002.

9. McKeown SJ, Newgreen DF and Farlie PG: Dlx2 over-expression regulates cell adhesion and mesenchymal condensation in ectomesenchyme. Dev Biol 281: 22-37, 2005.

10. Gordon CT, Brinas IM, Rodda FA, Bendall AJ and Farlie PG: Role of Dlx genes in craniofacial morphogenesis: Dlx2 influences skeletal patterning by inducing ectomesenchymal aggregation in ovo. Evol Dev 12: 459-473, 2010

11. Dai J, Kuang Y, Fang B, Gong H, Lu S, Mou Z, Sun H, Dong Y, Lu J, Zhang W, et al: The effect of overexpression of Dlx2 on the migration, proliferation and osteogenic differentiation of cranial neural crest stem cells. Biomaterials 34: 1898-1910, 2013.

12. Shibukawa Y, Young B, Wu C, Yamada S, Long F, Pacifici M and Koyama E: Temporomandibular joint formation and condyle growth require Indian hedgehog signaling. Dev Dyn 236: 426-434, 2007

13. Michikami I, Fukushi T, Honma S, Yoshioka S, Itoh S, Muragaki Y, Kurisu K, Ooshima T, Wakisaka S and Abe M: Trps1 is necessary for normal temporomandibular joint development. Cell Tissue Res 348: 131-140, 2012.
14. Oka K, Oka S, Sasaki T, Ito Y, Bringas P Jr, Nonaka K and Chai Y: The role of TGF-beta signaling in regulating chondrogenesis and osteogenesis during mandibular development. Dev Biol 303: 391-404, 2007.

15. Owtad P, Park JH, Shen G, Potres Z and Darendeliler MA: The biology of TMJ growth modification: A review. J Dent Res 92: 315-321, 2013.

16. Dai J, Wang J, Lu J, Zou D, Sun H, Dong Y, Yu H, Zhang L, Yang T, Zhang X, et al: The effect of co-culturing costal chondrocytes and dental pulp stem cells combined with exogenous FGF9 protein on chondrogenesis and ossification in engineered cartilage. Biomaterials 33: 7699-7711, 2012.

17. Kinumatsu T, Shibukawa Y, Yasuda T, Nagayama M, Yamada S, Serra R, Pacifici M and Koyama E: TMJ development and growth require primary cilia function. J Dent Res 90: 988-994, 2011.

18. Ochiai T, Shibukawa Y, Nagayama M, Mundy C, Yasuda T, Okabe T, Shimono K, Kanyama M, Hasegawa H, Maeda Y, et al: Indian hedgehog roles in post-natal TMJ development and organization. J Dent Res 89: 349-354, 2010.

19. Alappat S, Zhang ZY and Chen YP: Msx homeobox gene family and craniofacial development. Cell Res 13: 429-442, 2003.

20. Diamond E, Amen M, Hu Q, Espinoza HM and Amendt BA: Functional interactions between Dlx 2 and lymphoid enhancer factor regulate Msx2. Nucleic Acids Res 34: 5951-5965, 2006.

21. Iwata J, Hacia JG, Suzuki A, Sanchez-Lara PA, Urata M and Chai Y: Modulation of noncanonical TGF- $\beta$ signaling prevents cleft palate in Tgfbr2 mutant mice. J Clin Invest 122: 873-885, 2012.

22. Wang XD, Kou XX, He DQ, Zeng MM, Meng Z, Bi RY, Liu Y, Zhang JN, Gan YH and Zhou YH: Progression of cartilage degradation, bone resorption and pain in rat temporomandibular joint osteoarthritis induced by injection of iodoacetate. PLoS One 7: e45036, 2012.

23. Embree M, Ono M, Kilts T, Walker D, Langguth J, Mao J, Bi Y, Barth JL and Young M: Role of subchondral bone during early-stage experimental TMJ osteoarthritis. J Dent Res 90: 1331-1338, 2011

24. Jiao K, Zhang M, Niu L, Yu S, Zhen G, Xian L, Yu B, Yang K, Liu P, Cao X and Wang M: Overexpressed TGF- $\beta$ in subchondral bone leads to mandibular condyle degradation. J Dent Res 93: 140-147, 2014. 